\title{
A Tunnel Compress Scheme for Multi-Tunneling in PMIPv6-based Nested NEMO
}

\author{
Min-Soo Woo ${ }^{1}$, Hyo-Beom Lee ${ }^{2}$, Youn-Hee Han $^{3}$ and Sung-Gi Min ${ }^{4}$ \\ ${ }^{1}$ Dept. of Computer Science and Engineering, Korea University, Seoul, South Korea \\ ${ }^{2}$ Dept. of Computer Science and Engineering, Korea University, Seoul, South Korea \\ ${ }^{3}$ School of Internet-Media, Korea University of Technology and Education, Cheon-An, \\ South Korea \\ ${ }^{4}$ Dept. of Computer Science and Engineering, Korea University, Seoul, South Korea \\ e-mail:sgmin@korea.ac.kr
}

\begin{abstract}
In nested NEMO, a multi-tunneling causes a pinball routing problem. Several solutions proposed to solve the pinball routing problem in NEMO BSP cannot be used at PMIPv6-based NEMO due to different environment such as no route optimization with $C N$. We propose a tunnel compress scheme for multi-tunneling in PMIPv6-based NEMO. The scheme consists of two parts: the first part is an interdomain or wired Internet part. The other is an intra part of nested mobile networks. In the inter-domain part, single IP-in-IP tunnel is created by connecting an innermost entry point with an outermost exit point in original multi-tunnels. As the same way used in the inter-domain part, single IP-in-IP tunnel is created from the outermost exit point and an innermost exit point in original multi-tunnels. In the proposed scheme, IP-in-IP encapsulated packets are forwarded using host-based routing without modifying the outer header. The information to compress multi-tunnels is piggybacked at the PMIPv6 signaling.
\end{abstract}

\section{Keywords}

PMIPv6, NEMO, nested mobile networks, pinball routing problem

\section{I.INTRODUCTION}

With rapid adaptation of mobile devices, the need to be connected to Internet during traveling has become high. Several transportations support the need and mobile networks become a kind of common network environments. To support Network Mobility (NEMO), NEMO Basic Support Protocol (NEMO BSP) [1] is proposed by IETF working group. NEMO BSP is based on Mobile IPv6 (MIPv6) [2]. The access router on a moving vehicle is called a Mobile Router (MR). The MR aggregates all traffic to MNs connected to the mobile network and it forwards the aggregated traffic to the MR's Home Agent (HA). A mobile network can be attached other mobile network, and as such way multiple mobile networks can be nested. When mobile networks are nested, NEMO BSP in each mobile network can operate independently. However, multi-tunnels in nested mobile networks are created between Correspondent Node $(\mathrm{CN})$ and Mobile Node (MN) because each mobile network creates a tunnel. This multi-tunneling has two problems. One is an encapsulation overhead. Whenever a tunneling is applied, all packets must have additional header, which requires data shift in packet buffer and fragmentation of packets if the size of packets is almost the same as that of Maximum Transfer Unit (MTU). The other is 
multiple packet forwarding, called a pinball routing problem [4], causing additional packet delay and workload while packets are forwarded between HAs.

Previous schemes such as [5][6] were proposed to solve multi-tunneling problems. However, these MIPv6-based schemes cannot be used at Proxy Mobile IPv6 (PMIPv6) [3] based on NEMO. PMIPv6 does not provide Route Optimization (RO) scheme with a CN. A MN in PMIPv6 does not participate in mobility signaling and does not have Care-of Address (CoA).

In this paper, we propose a Tunnel Compress Scheme (TCS) to solve multi-tunneling problems at PMIPv6-based NEMO. The TCS reduces multiple tunneling overhead. Multiple tunnels are partitioned into two parts. The first part is an inter-domain or wired Internet part, and the second part is a wireless or intra part of nested mobile networks. The partitioning is done at the outermost exit point of multi-tunnels. Fig. 1 shows two parts of PMIPv6-based NEMO networks. In wired part, CN, LMAs and MAG4 are connected by wired cables. However, in wireless part, MAGs and MN are wireless mobile nodes. In nested NEMO, the outermost exit point acts as the first access gateway to wired Internet. In Fig. 1, the outermost exit point is MAG4.

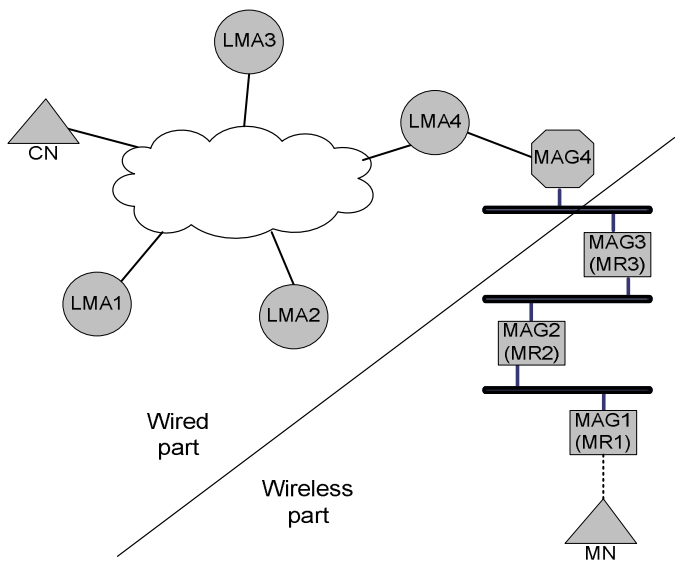

Figure 1.Pinball routing problem

The TCS compresses the multi-tunnels between the innermost entry point and the outermost exit point with the single IP-in-IP tunnel. Then, the packet forwarding from the outermost exit point to the innermost exit point is done by host-based routing, which can be applicable to Local Mobility Management (LMM) protocols. As PMIPv6 is one of LMM protocols and Mobile Access Gateways (MAGs) forwards packets to MNs by host-based concepts, the host-based routing can be easily integrated into PMIPv6 components.

\section{I1. RELATED WORKS}

Several schemes have been proposed to solve pinball routing problem. Reverse Routing header (RRH) [5] defines a new routing header as extension header of IPv6. The routing header records optimized path from a MN to a HA. In this approach, packets are forwarded to the MN using source routing based on path information of the routing header. RRH creates only single IP-in-IP tunnel, and forwards packets from MN's HA to access router attached to nested mobile networks. However, header modifications must be performed in every packet passed through the tunnel. Furthermore, RRH has a security problem. To solve problems of RRH, Nested Path Information (NPI) [7] was proposed. In NPI, nested path information is included in a Route Advertisement (RA) message. If a MN receives this RA message, the MN immediately sends a 
Binding Update (BU) message with nested path information to inform a HA. However, when nested mobile networks move and are attached to another access router, new nested path information will be spread to nested mobile networks. Every MR and MN receiving this information immediately sends a BU message with new information. In this case, binding update storm can be caused.

Access Router Option (ARO) [6] solves the pinball routing problem using RO scheme of MIPv6. In nested mobile networks, a HA which a MR is registered is treated as a CN from the perspective of an upper MR of the MR. The upper MR performs RO procedure with the HA. After completing RO procedure, packets are forwarded from the HA to the upper MR directly. ARO is simple and requires minimum changes in existing NEMO BSP. However, a long convergence time is needed. Also, to find a Top Level of MR (TLMR) of nested mobile networks, binding caches should be looked up recursively. If degree of nesting is increased, convergence and search times are getting longer.

To solve the pinball routing problem Hierarchical MIPv6 (HMIPv6) approaches are proposed. In HMIP based Route Optimization (HMIP-RO) [8] and Hierarchical Route Optimization (HRO) [9], a TLMR of nested mobile networks acts a role of Mobility Anchor Point (MAP) of HMIPv6. Every MR in nested mobile networks creates Regional CoA (RCoA) using Home Address (HoA) prefix of TLMR and informs to HA. After receiving information, HA forwards packets to the TLMR directly. Through this process, routing optimization is accomplished. However, in HMIP-RO, multi-tunnels may be caused in intra part of nested mobile networks. Besides, binding update storm can occur in HMIP-RO as the same as in NPI. In case of HRO, binding registration time and overhead of MAP are increased because binding caches are searched recursively.

Route Optimization Using Tree Information Option (ROTIO) [10] is another approach. Extended TIO (xTIO) which is extension of Tree Information Option (TIO) is used in ROTIO. ROTIO supports seamless handoff when nested mobile networks are attached another AR. However, to support seamless handoff, packets are passed through previous point which MR is attached to. Hence a routing path is getting longer. Moreover, if handover occurs dynamically, communication may be disrupted.

\section{The Tunnel Compress Scheme}

\section{A. Nested NEMO in PMIPv6-based network}

NEMO can be supported by PMIPv6 [11]. Fig. 2 shows the nested NEMO in PMIPv6-based network. As in nested NEMO, this orthogonal relationship to support nested node mobility causes pinball problem. There are four overlaid PMIPv6 networks to support depth 3 nested mobile networks. The LMA4 and the MAG4 support the mobility of the MR3. The MR3 itself must be a MAG to support nested nodes mobility. As the MN does not involve in the mobility management, the MAG1 registers the MN at the LMA1 on behalf of the MN. All traffic bound to the MN is tunneled to the MAG1 by the LMA1. Same as MAG1, All traffic is tunneled to the MAG2 and MAG3 using each independent tunnel. Besides, the other tunnel is created between LMA4 and MAG4 by PMIPv6 specification. Fig. 3 shows the multi-tunnels to forward packets to the MN for the LMA1. 
The PMIPv6 network consists of wired part and wireless part. All mobile networks belong to the wireless network part. The forwarding multi-tunnels have the growing phase and the shrinking phase as well. The growing phase ends at the MAG4, which is the last access gateway of wired part as

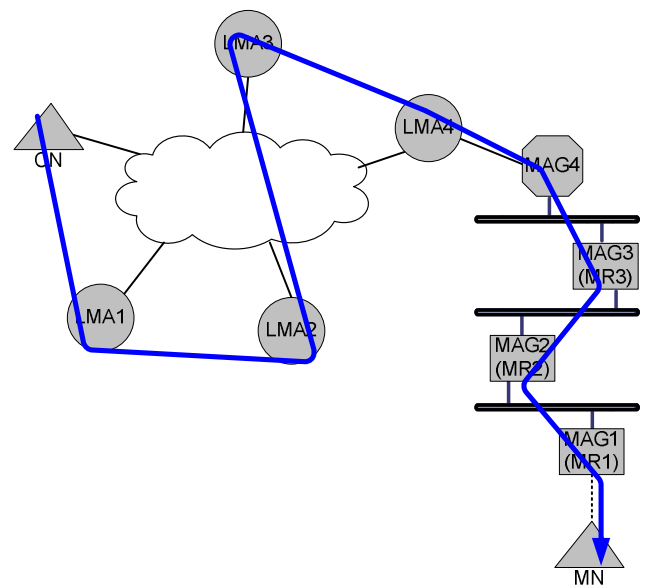

Figure 2. Pinball routing problem

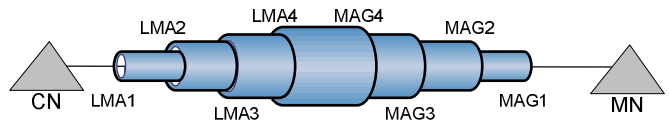

Figure 3. Multi-tunnels from a $\mathrm{CN}$ to a $\mathrm{MN}$

well as the first gateway to the wireless part. The purpose of the growing phase is aggregation of all traffic bound to the nested mobile network to avoid binding update storm by providing mobility transparency to MNs under the mobile networks. However, the aggregated traffic must be forwarded to the right access gateway which forwards the traffic into the mobile networks. Therefore the growing phase has two purposes: one is the traffic aggregation and the other is routing the aggregated traffic to the first gateway to the wireless network part. The traffic aggregation is done by the innermost tunnel of the multi-tunnels and the routing is done by outer layers of the multi-tunnels.

The shrinking phase starts from MAG4 and it ends at the MAG1. The outermost layer of the multi-tunnels is eliminated when the outer tunnel reaches its destination MAG. In PMIPv6 MAGs do the host-based mobility handling for MNs. They send specific RA for a MN and forward packets based on MNs' home network prefixes; MAGs have the host-based routing functionality. When the MAG4 terminates the outer most tunnel of the multi-tunnel, it gets the next layer packet which has the MAG3 as the next destination. As the MAG4 does host-based routing, it knows how to route the packets having the MAG3 as the next destination. Therefore the purpose of the next layer tunnel is to route the forwarded packets to MAG3. The shrink phase of the multi-tunnel acts as routing packets from MAG4 to MAG1.

\section{B. The Tunnel Compress Scheme}

The TCS compresses the multi-tunnels between the innermost entry point of the multitunnels and the outermost exit point of the multi-tunnels into single IP-in-IP tunnel. The innermost entry point of the multi-tunnels encapsulates all packets toward the innermost exit 
point of the multi-tunnels with IP-in-IP encapsulation and the encapsulated packets have the outermost exit point of the multi-tunnels as their destination rather than the innermost exit point of the multi-tunnels. In our example, all packets bound to the MAG1 are encapsulated with IPin-IP encapsulation and their destination becomes the MAG4 instead of the MAG1.

The TCS uses the MAG's host-based routing functionality from the outermost exit point to the innermost exit point of the multi-tunnels. Rather than MAGs decapsulate tunneled packets, it installs the host-based routing entry into their routing entry. Because the tunnel itself carries an aggregated traffic, installing new routing entries for MAGs coincides with the concept of IP routing. The intermediate MAGs of the multi-tunnels forward the tunneled packets rather than decapsulate them.

TCS extends Proxy Binding Update (PBU) message. Fig. 4 shows the extended PBU format. The PBU message has new "T" flag and TCS option. The "T" flag represents that the initiating MAG wants to use the TCS. The TCS option records the multi-tunnel information. Each intermediate MAG adds its LMA information to the TCS option. TCS option has records of $<$ LMA, MAG > pair.

When a mobile network is attached to other mobile network, the MAG in NEMO network, which the mobile network will be attached, generates a PBU. The MAG refers

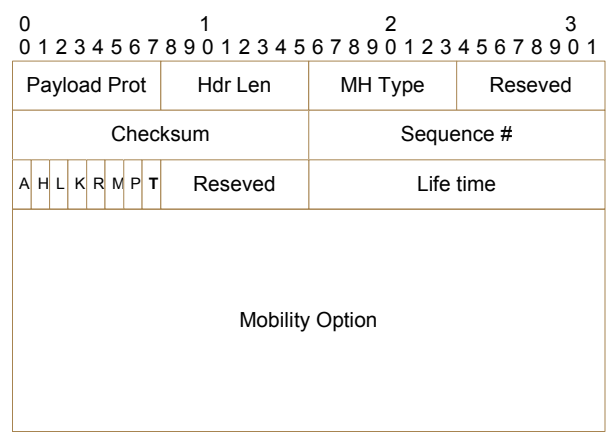

(a) Mobility header

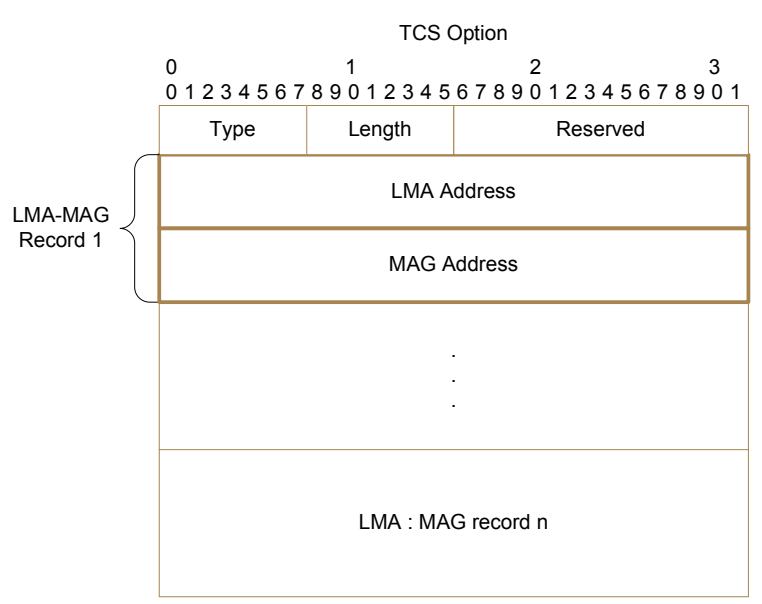

(b) TCS option 
AAA server to get attached node information. The information includes home LMA address of the attaching node. The MAG creates two LMA_MAG records: one for the attaching node and the other for itself. The PBU is forwarded to the upper MAG. The upper MAG information is taken from RA. When the upper MAG receives the PBU with "T" flag set, the MAG appends its LMA_MAG record to the PBU. If the received MAG is not the mobile router, the MAG knows that it is the top MAG of the path. The top MAG forwards the LMA of the second record of TCS option. When the LMA of the second record receives the PBU, it records the first LMA, which is the inner tunnel LMA, and it forwards the PBU to the first LMA. The recording procedure is the same as inter-roaming draft [12]. The recording inner tunnel LMA is very important to repair the multi-tunnels when intermediate MAG (MR) handovers to other PMIPv6.

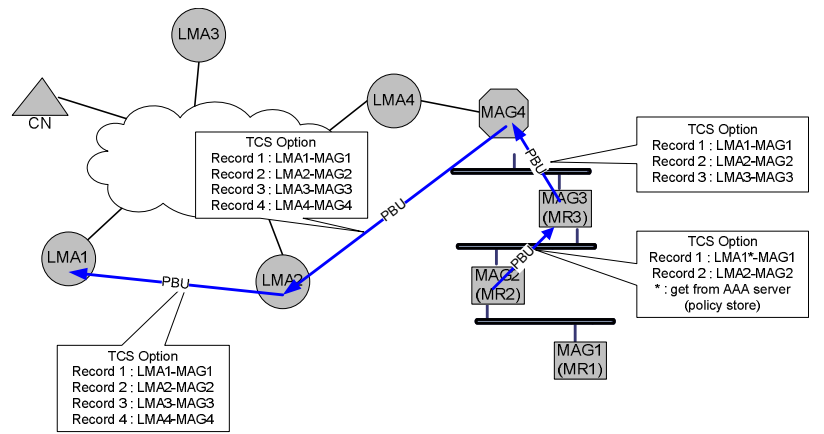

Figure 5. PBU flow when MAG1 is attached to MAG2

Fig. 5 shows the PBU flow when the MAG1 is attached to MAG2. When the MAG2 detects the attachment of the MAG1, it creates a PBU and adds a TCS option. It forwards the PBU to the MAG3. When the MAG3 receives the PBU, it appends its <LMA3, MAG3> record to the TCP option and it creates the routing entry for the MAG1. Then it forwards the PBU to the MAG4. When the MAG4 receives the PBU, it also appends its <LMA4, MAG4> record to the TCS option and creates routing entry for the MAG1. The MAG4 is not the mobile, so it knows it is the top MAG of the new path. It must create TCS tunnel entry for the MAG1, and then it forwards the PBU to the LMA2 directly because the path to the LMA2 was created already when the MAG2 is registered. When the LMA2 receives the PBU, it records the inner LMA (LMA1) and forwards the PBU to the LMA1. When the LMA1 receives the PBU, it creates the TCS tunnel entry. The tunnel entry is composed by the TCS option. The MAG of the last LMAMAG record in the TCS option becomes the destination of the TCS tunnel. After creating the tunnel entry, the LMA1 sends a PBA to the LMA2. The LMA2 forwards MAG4 and the MAG4 forwards the PBA to the MAG2 via the MAG3.

Fig. 6 shows the routing tables and the TCS tunnel table. New entries created by the PBU are highlighted. The MAG4 looks up the tunneling table to find out whether a packet uses the TCS tunnel or not when the packet arrives at the MAG4. If so, the MAG4 terminates the tunnel and encapsulates the packet. The encapsulated packet has the final destination of the original multitunnels as the destination of the outer header. Intermediate MAGs, MAG3 and MAG2, just forwards the encapsulated packet. The forwarding routing entry is created by receiving a PBU when the MAG1 is attached to the MAG2.

After completing binding registration process with the TCS option, packets are forwarded along the path as shown in Fig. 7. If the $\mathrm{CN}$ sends packets to the MN, the LMA1 intercepts packets. Then the LMA1 encapsulates packets using TCS tunnel entry. This encapsulated packet 
International Journal of Wireless \& Mobile Networks (IJWMN) Vol.2, No.4, November 2010

is the same as packet format of the LMA1 of Fig. 7. Also the left tunnel of Fig. 8 shows the IPin-IP tunnel of outer header when the LMA1 encapsulates packets. Destination of outer

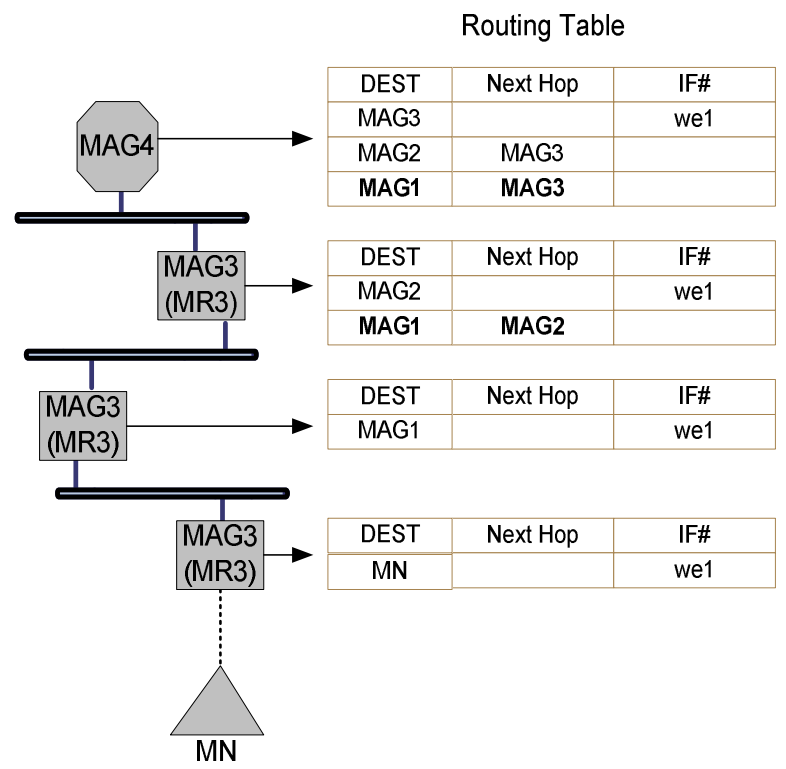

Figure 6. Routing table and TCS tunneling table

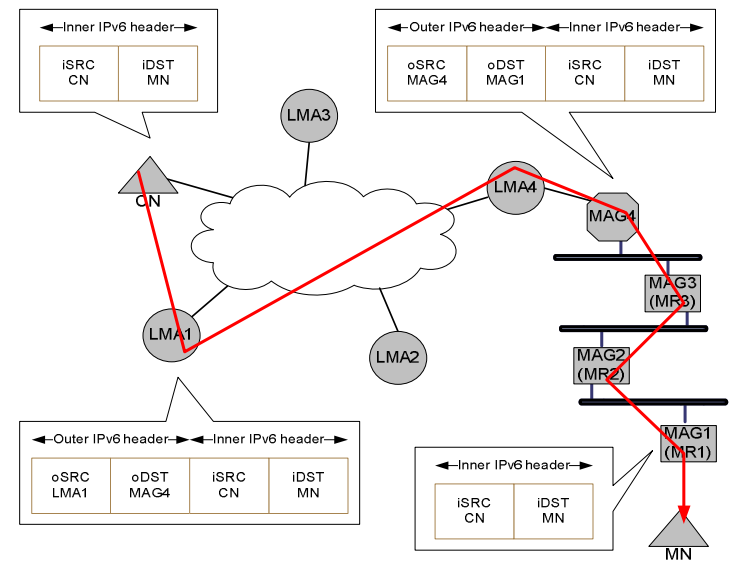

Figure 7. A routing path in TCS

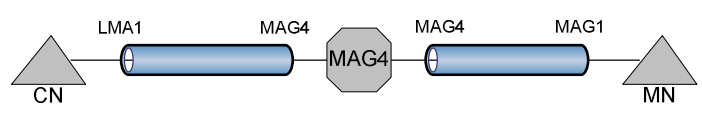

Figure 8. Two single IP-in-IP tunnels in TCS

header is the MAG4, so packets are forwarded the MAG4 directly. At this time, optimization of routing path is performed. 
The MAG4 checks TCS tunneling table whether packets are belonged to TCS tunnel or not. If so, the MAG4 decapsulates the outer header, and then encapsulates packets once again with new IP-in-IP tunnel header. This tunnel is the same as shown the right tunnel of Fig. 8. Hence Packets are forwarded into nested mobile networks. The MAG3 and the MAG2 forward packets according to routing tables without modification of header. After receiving packets, MAG1 decapsulates outer header and then it sends to the MN. In this process, routing path can be optimized through the first tunnel, and packet is forwarded in nested mobile networks just using host-based routing.

\section{Movement of Nested mobile networks}

When nested mobile networks are moved, several tunnels are affected by the event. All communication using these tunnels is disrupted because the TCS tunnel consists of two tunnel segments and each tunnel segment is terminated at the outermost exit point. In this case, the destination of two tunnel segments and host-routing tables must be updated. The outermost exit point must send two PBUs simultaneously; one for updating TCS information of LMAs, and the other for updating MAGs.

Fig. 9 shows the example of two simultaneous PBUs when nested mobile networks move into MAG3_1.

As the MAG3 returns its home network, the destination of these tunnels must be changed from the MAG4 to the MAG3_1. When the MAG3_1 detects movement of the MAG3, it creates the PBU with a TCS option. The PBU is forwarded to the LMA3. As the PBU has the "T" flag, the LMA3 knows that nested mobile networks have at least one another LMA to be notified. The information was recorded in the LMA3 when the MAG2 attached to the MAG3. The
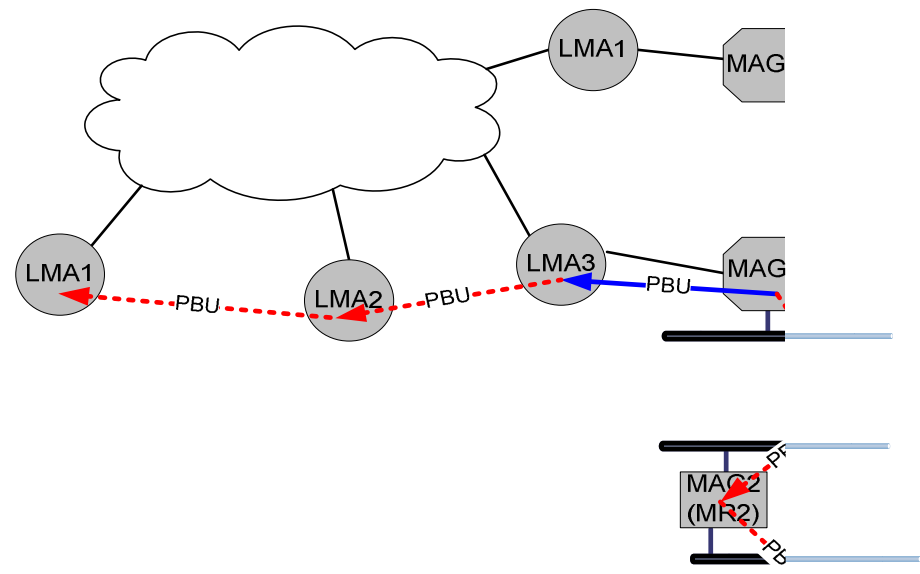

Figure 9. PBU flow when nested mobile networks move

LMA3 forwards the PBU to the LMA2. As the same reason, the LMA2 forwards the PBU to the LMA1. The LMA1 updates its TCP tunnel entry and sends the PBA to the LMA2. This forwarding continues until the PBA arrives at the MAG3_1. 
The MAG3_1 sends the PBU to the MAG3, and the PBU is forwarded until it arrives at the MAG1. MAGs receiving the PBU update the reverse tunnel destination from MAG4 to MAG3_1 as well as routing table entry if necessary.

\section{EXPERIMENTS}

We perform simulation the proposed TCS using NS-2 network simulator. A simulation topology and environments are set up the same as Fig. 1. We measure transmission times from a $\mathrm{CN}$ to a $\mathrm{MN}$, and packet sizes when a packet is passed through each network component. We compare TCS with PMIPv6-based NEMO through measured time and size.

Fig. 10 shows a transmission time in each packet. In TCS, the packet transmission time is decreased rather than the PMIPv6-based NEMO. This means that packets do not go through LMAs such as LMA2 and LMA3. Therefore, TCS performs route optimization well.

Fig. 11 shows the size of a packet at each network component to compare the count of encapsulation between PMIPv6-based NEMO and TCS. We set a data packet size to 1000 bytes. In PMIPv6-based NEMO, the packet size has gradually increased since the packet is reached to MAG4, and then has decreased by degrees. It is noticed that a packet is encapsulated in each LMA and decapsulated in each MAG. We note that the MAG4 has bigger packet size than the LMA4, because additional information is appended to packets for transmitting in wireless section. On the other hand, packet size does not increase at all from LMA4 to the MAG2 if we consider the additional packet overhead in wireless section as the same as in PMIPv6-based NEMO. We also note that the packet size in LMA2 and LMA3 cannot be drawn because packets never go through these LMAs. From this result, we know that packets are encapsulated once in the proposed TCS, and therefore tunneling overhead decreases rather than PMIPv6-based NEMO.

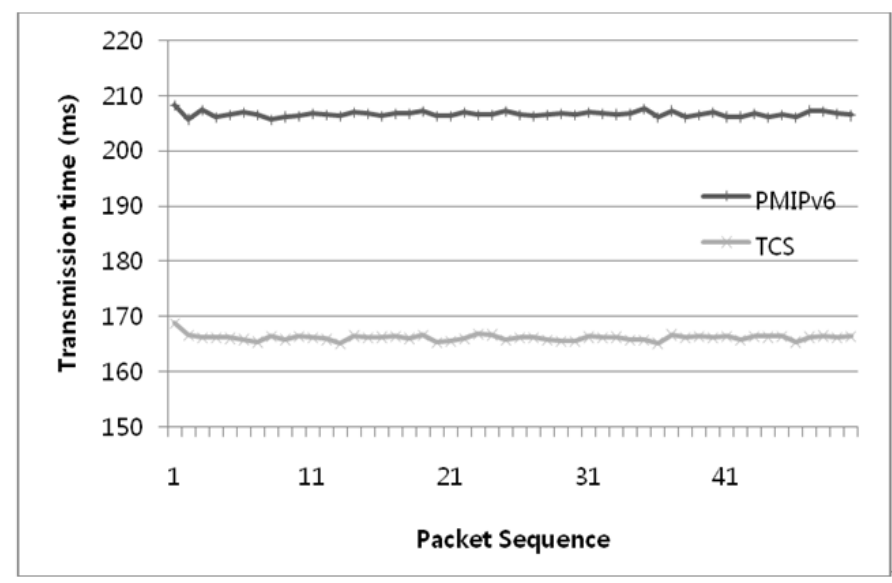

Figure 10. Transmission time from a $\mathrm{CN}$ to a $\mathrm{MN}$ in each packet 
International Journal of Wireless \& Mobile Networks (IJWMN) Vol.2, No.4, November 2010

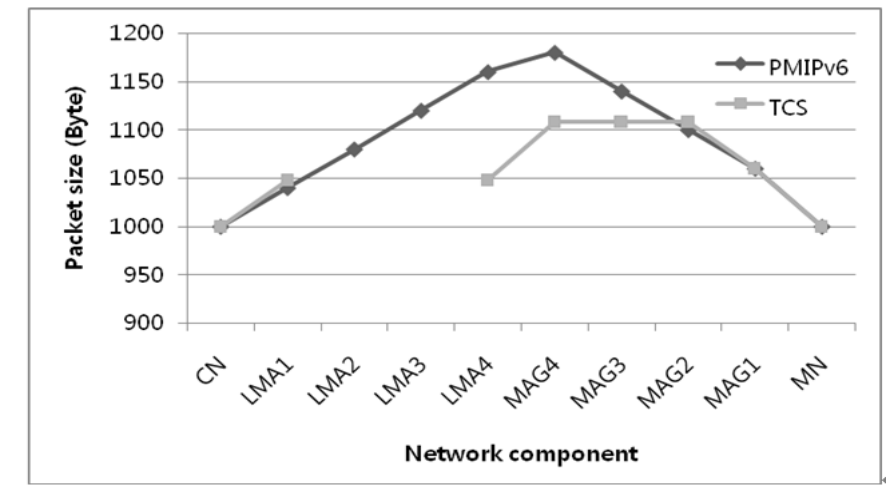

Figure 11. Packet size when a packet leaves each network component

\section{V.CONCLUSION}

To solve multi-tunneling problems in nested NEMO, several MIPv6-based solutions are proposed. However, these approaches cannot be used at PMIPv6-based NEMO due to different environment such as no route optimization with $\mathrm{CN}$.

In this paper, we proposed the TCS to solve multi-tunneling problems at PMIPv6-based NEMO. The pinball routing problem can be solved by optimizing the routing path in TCS. Furthermore, when nested mobile networks are attached to another MAG, communication is continuously kept up. Simulation results show that the routing path can be optimized by skipping intermediate LMAs in TCS and tunneling overhead also decreases.

\section{References}

[1] V. Devarapalli, R. Wakikawa, A. Petrescu, and P. Thubert, "Network Mobility (NEMO) Basic Support Protocol," RFC 3963, Jan. 2005.

[2] D. Johnson, C. Perkins, and J. Arkko, "Mobility support in IPv6," RFC 3775, Jun. 2004.

[3] S. Gundavelli, K. Leung, V. Devarapalli, K. Chowdhury and B. Patil, "Proxy Mobile IPv6," RFC 5213, Aug. 2008.

[4] C. Ng, P. Thubert, H. Ohnishi, and E. Paik, "Taxonomy of Route Optimization models in the NEMO Context," Internet Draft, draft-thubert-nemo-ro-taxonomy-04, Feb. 2005.

[5] P. Thubert, and M. Molteni, "IPv6 Reverse Routing Header and its application to Mobile Networks," Internet Draft, draft-thubert-nemo-reverse-routing-header-07, Feb. 2007.

[6] C. Ng, and J. Hirano, "Securing Nested Tunnels Optimization with Access Router Option," Internet Draft, draft-ng-nemo-access-router-option-01, Jul. 12, 2004.

[7] J. Na, C. Kim, S. Lee, H. Kang, and C. Koo, "Secure Nested Tunnels Optimization using Nested Path Information," Internet Draft, draft-na-nemo-nested-path-info-00, Sep. 2003.

[8] H. Ohnishi, K. Sakitani, and Y. Takagi, "HMIP based Route optimization method in a mobile network," Internet Draft, draft-ohnishi-nemo-ro-hmip-00.txt, Oct. 2003.

[9] L. Dongkeun, K. Keecheon, and K. Moonhae, "Hierarchical Route Optimization for Nested Mobile Network," 18th International Conference on Advanced Information Networking and Application (AINA’04) pp. 225-229, Mar. 2004.

[10] C. Hosik, K. Taekyoung, and C. Yanghee, "Route Optimization Using Tree Information Option for Nested Mobile Networks," IEEE Journal on Selected Areas in Communications, vol. 24, no. 9, Sep. 2006.

[11] R. Wakikawa, S. Gundavelli, and Y. Zhao, "Network Mobility Support for Proxy Mobile IPv6," Internet Draft, draft-wakikawa-netext-pmip6-nemo-support-00.txt, Apr. 2009.

J-H. Na, S. Park, J-M. Moon, S. Lee, E. Lee, and S-H. Kim, "Roaming Mechanism between PMIPv6 Domains,", Internet Draft, draft-park-netlmm-pmipv6-roaming-01, Jul. 2008. 